\title{
Mediterranean diet adherence during pregnancy and fetal growth: INMA (Spain) and RHEA (Greece) mother-child cohort studies
}

\author{
Leda Chatzi ${ }^{1 *}$, Michelle Mendez ${ }^{2,3}$, Raquel Garcia ${ }^{2,3}$, Theano Roumeliotaki ${ }^{1}$, Jesús Ibarluzea ${ }^{3,4}$, \\ Adonina Tardón ${ }^{3,5}$, Pilar Amiano ${ }^{3,4}$, Aitana Lertxundi ${ }^{3,4,6}$, Carmen Iñiguez ${ }^{3,7}$, Jesus Vioque ${ }^{3,8}$, \\ Manolis Kogevinas ${ }^{2,3,9,10}$, Jordi Sunyer ${ }^{2,3,10,11}$ on behalf of the INMA and RHEA study groups \\ ${ }^{1}$ Department of Social Medicine, Faculty of Medicine, University of Crete, Heraklion, Greece \\ ${ }^{2}$ Centre for Research in Environmental Epidemiology (CREAL), Barcelona, Spain \\ ${ }^{3}$ CIBER Epidemiologia y Salud Pública (CIBERESP), Spain \\ ${ }^{4}$ Public Health Division of Gipuzkoa, Instituto Investigation BioDonostia, Basque Government, Spain \\ ${ }^{5}$ Universidad de Oviedo, Spain \\ ${ }^{6}$ University of Basque Country, EHU-UPV, Spain \\ ${ }^{7}$ Centre Superior de Investigation en Salud Publica (CSISP), Valencia, Spain \\ ${ }^{8}$ Departmento Salud Publica, Campus San Juan, Universidad Miguel Hernandez, Elche-Alicante, Spain \\ ${ }^{9}$ National School of Public Health, Athens, Greece \\ ${ }^{10}$ Municipal Institute of Medical Research, Barcelona, Spain \\ ${ }^{11}$ Universitat Pompeu Fabra, Barcelona, Spain
}

(Received 5 October 2010 - Revised 5 April 2011 - Accepted 8 April 2011 - First published online 29 June 2011)

\begin{abstract}
Dietary intake of specific nutrients or food groups during pregnancy could influence fetal growth, but scant evidence is available on effects of dietary patterns. The aim of this study was to evaluate the impact of Mediterranean diet (MD) adherence during pregnancy on fetal growth in two population-based mother-child cohorts in Spain and Greece. We studied 2461 mother-newborn pairs from the Spanish multi-centre 'INMA' study (Atlantic area: INMA-Atlantic; Mediterranean area: INMA-Mediterranean), and 889 pairs from the 'RHEA' study in Crete, Greece. Maternal diet during pregnancy was assessed by FFQ and MD adherence was evaluated through an $a$ priori score. Fetal growth restriction was based on a customised model, and multivariate log-binomial and linear regression models were used to adjust for several confounders. MD scores differ significantly between the cohorts with women in INMA-Atlantic reporting higher intakes of fish and dairy products, while women in the Mediterranean area reported higher intakes of cereals, vegetables and fruits. Women with high MD adherence had a significantly lower risk of delivering a fetal growth-restricted infant for weight (risk ratios: 0.5; 95\% CI 0.3, 0.9) in the INMA-Mediterranean cohort. Stratified analysis by smoking revealed that higher MD adherence increased birth weight and birth length in smoking mothers, whereas this effect was not apparent in non-smoking mothers. The results of the present study show that several types of MD exist across European Mediterranean regions. High MD adherence may modify the detrimental effect of smoking on birth size, but overall effects of diet were not universal for the studies in this analysis.
\end{abstract}

Key words: Mediterranean diet: Pregnancy: Fetal growth: Smoking

A substantial body of epidemiological evidence suggests that a poor in uterus environment elicited by maternal dietary or placental insufficiency may 'programme' susceptibility in the fetus to later development of CVD and metabolic disease. Fetal growth restriction is an important predictor of a baby's health and chances of survival, both in the long term and in the short term ${ }^{(1)}$. Epidemiological studies suggest that low-birthweight (BW) infants have a higher risk later in life for developing CVD, and poor cognitive and neurological development ${ }^{(2-4)}$.

Although few studies have examined the role of diet during pregnancy in offspring growth in Western industrialised countries, several aspects of maternal diet are hypothesised to influence fetal growth, including low glycaemic load diets $^{(5)}$, fruit and vegetable intake ${ }^{(67)}$, fish intake ${ }^{(8-13)}$, dairy products intake ${ }^{(14)}$ and some vitamins and antioxidants ${ }^{(15,16)}$,

Abbreviations: BL, birth length; BW, birthweight; FlGR, fetal length growth restriction; FwGR, fetal weight growth restriction; HC, head circumference; INMA, Infancia y medio Ambiente; MD, Mediterranean diet.

*Corresponding author: L. Chatzi, fax +30 2810 394606, email lchatzi@med.uoc.gr 
with conflicting results. These studies may fail to account for the interactions between nutrients, and they do not take into consideration that some nutrients are inter-correlated ${ }^{(17)}$. Thus, interest has shifted to place greater emphasis on dietary patterns that represent a broader picture of food and nutrient consumption and may therefore be more predictive of disease risk. The evaluation of dietary patterns in pregnant populations is rare. Results from the Danish National Birth Cohort revealed that a diet in pregnancy, rich in red and processed meat and high-fat dairy products, was associated with increased risk for small-for-gestational-age infants ${ }^{(18)}$, whereas women following a Mediterranean dietary pattern had lower risk for preterm birth $^{(19)}$. A recent study from Valencia (part of the Spanish Infancia y Medio Ambiente (INMA) birth cohort) has shown that women with the highest quality diet in the first trimester of pregnancy, based on the Alternate Healthy Eating Index, had a significantly lower risk of delivering a fetal growthrestricted infant for weight ${ }^{(20)}$

The evaluation of the potential association of the Mediterranean diet (MD) with fetal growth fits well in the context of assessing the overall effect of the adherence to a dietary pattern on the occurrence of a disease. In general, the MD is characterised by elevated intake of plant foods such as fruits and vegetables, bread and cereals (primarily wholegrain), legumes and nuts. Low-to-moderate amounts of dairy products and eggs, and only small amounts of red meat are included in the diet. This dietary pattern is low in saturated fatty acids, rich in carbohydrates, fibre and antioxidants, and has a high content of MUFA, and $n$ - 3 PUFA, which are primarily derived from olive oil and fish intake ${ }^{(21)}$.

The objective of this study was to determine the association between MD in pregnancy and fetal growth in two large population-based mother-child cohorts in the Mediterranean area: the INMA study, which is a collaborative mother and child cohort that was established in Spain in $2003^{(22)}$, and the RHEA study in Crete, Greece, a population-based pregnancy cohort initiated in Crete in $2007^{(23)}$.

\section{Methods}

\section{Study population}

The INMA cohort. In this paper, we analysed data obtained prospectively from pregnant women enrolled in the INMA study from the Atlantic area (INMA-Atlantic: Guipuzcoa (Basque Country), Asturias), and the Mediterranean area (INMA-Mediterranean: Valencia, Sabadell (Catalonia)). All pregnant women were recruited at their first routine antenatal care visit in the main public hospital or health centre of reference for defined geographical areas. The recruitment period was from February 2004 to June 2005 in Valencia, from April 2006 to January 2008 in Guipuzcoa, from July 2004 to July 2006 in Sabadell and from May 2004 to June 2007 in Asturias. Questionnaires regarding health behaviours, socio-demographic characteristics, pregnancy history and other factors were administered by trained interviewers at recruitment, during the third trimester and at delivery. There were 2616 women who agreed to participate and met the inclusion criteria (being at least 16 years old, having a singleton pregnancy and not following any programme of assisted reproduction). We included only those women who had completed an FFQ in the first trimester ( $n$ 2585), and for whom complete information on reproductive outcomes ( $n$ 2481) was available. A total of twenty additional women were excluded from the analysis because of implausible values for total energy intake (outside the range of $4184-16736 \mathrm{~kJ} /$ d) ${ }^{(24)}$. Thus, the final analysis was based on 2461 pregnant women (777 in Valencia, 604 in Guipuzcoa, 610 in Sabadell and 470 in Asturias). The study was conducted according to the guidelines laid down in the Declaration of Helsinki and all procedures involving human subjects were approved by the ethics committee of the Institut Municipal d'Investigació Mèdica of Barcelona and by the ethics committees of the hospitals involved in the study. Written informed consent was obtained from all women participating in the cohort.

The RHEA cohort. The 'RHEA' project is a mother-child study that examines prospectively, a population-based cohort of pregnant women and their children, in the prefecture of Heraklion, Crete ${ }^{(23)}$. Female residents (Greek and immigrants) who became pregnant during the 12-month period from February 2007 until February 2008 were contacted and asked to participate in the study. The first contact was made at the time of the first major ultrasound examination, about week 15 of gestation or before. Face-to-face structured questionnaires together with self-administered questionnaires and medical records were used to obtain information on several dietary, environmental and psychosocial exposures during pregnancy and early childhood. The study was conducted according to the guidelines laid down in the Declaration of Helsinki and all procedures involving human subjects were approved by the ethics committee of the University Hospital in Heraklion, Crete, Greece. Written informed consent was obtained from all women participating in the study.

During the study recruitment period, 1765 eligible women were approached, 1610 (91\%) agreed to participate and 1317 (82\%) were followed up till delivery. Only singleton pregnancies were included in this analysis ( $n$ 1281). Women who experienced spontaneous or induced abortions ( $n$ 51) or gave birth to stillborn infants $\left(\begin{array}{ll}n & 2\end{array}\right)$ were excluded as were those women with incomplete information on reproductive outcomes ( $n$ 32) for any of the following reasons: change of address or delivery outside Heraklion prefecture, and/or incomplete or missing medical records. A total of 959 (80\%) participants provided complete questionnaire data on diet during pregnancy and reproductive outcomes. A total of seventy women were excluded from the analysis because of implausible values for total energy intake (outside the range of $4184-16736 \mathrm{~kJ} / \mathrm{d})^{(24)}$. Hence, a cohort of 889 women was available for this analysis.

\section{Dietary assessment}

INMA cohort. In the INMA cohort an FFQ was administered by trained interviewers, with 100 food items to assess usual food and nutrient intakes during the first trimester of pregnancy. The questionnaire asked how often, on average, 
participants had consumed a particular amount of a specific type of food from the last menstrual period until the time of the interview (mean 13.8 (SD 2.5) weeks of gestation). The FFQ was an adapted version of Willett's questionnaire ${ }^{(25)}$ that was developed and validated for use among adults living in Spain ${ }^{(26)}$. Standard units or reference serving sizes were specified for each food item. The questionnaire had nine possible intake frequency categories, ranging from 'never or less than once per month' to ' 6 or more times per day.' The intake frequency for each food item was converted to the average daily intake for each participant. Nutrient values were primarily obtained from the US Department of Agriculture foodcomposition tables and other published sources ${ }^{(20)}$. The nutrient intakes were calculated by multiplying the frequency of consumption for each food item by the nutrient composition of the portion size specified on the FFQ and by summing across all foods to obtain a total nutrient intake for each individual.

RHEA cohort. The RHEA FFQ was administered in mid pregnancy (14th-18th week of gestation) assessing dietary habits over pregnancy. This is a semi-quantitative questionnaire, containing 250 food items with the following components: intake frequency, dietary supplements, information on organic food consumption, different types of vegetarianism, dietary changes due to pregnancy, conditions of pregnancy that could affect diet and food intake (nausea, vomiting, etc.). The questions were hierarchical from general to more specific questions. For each food item, participants were asked about both the frequency of consumption and the average portion size. The exact frequency of consumption was given per day, per week and/or per month, depending on the food item. Photographs were used to visualise small, medium and large portion sizes for each food item and respondents had to choose one out of three pictures.

The intake frequency for each food item was converted to the average daily intake for each participant. For complex items (such as mixed dishes), standard recipes were used when available, otherwise new recipes that matched the description of the food were made and the intake of foods from different items was aggregated. Individual portion sizes and recipes were used to calculate nutrients intake based on the UK food tables (McCance and Widdowson's The Composition of Foods, 5th ed., plus nine supplements. London: HMSO). The food consumption data were analysed by a software developed at the Department of Applied Information Technology and Multimedia, TEI-Crete, Heraklion, Crete, Greece.

Mediterranean diet during pregnancy. To evaluate adherence to an MD during pregnancy, we used a scale applied in a large cohort study (European Prospective Investigation into Cancer and Nutrition; EPIC) in adults ${ }^{(27)}$. For beneficial components (vegetables, legumes, fruits and nuts, cereals, fish and seafood, dairy products), women whose consumption was below the median (cohort-specific median) were assigned a value of 0 , and women whose consumption was at or above the median were assigned a value of 1 . For components presumed to be detrimental (meat, including all types of meat), women whose consumption was below the median were assigned a value of 1 , whereas women whose consumption was at or above the median were assigned a value of $0^{(27)}$.
For fat intake (the eighth food category), we used the ratio of daily consumption of monounsaturated lipids to saturated lipids ${ }^{(27)}$. Because the index had been developed for adults and the present study population involved pregnant women, we presumed dairy products to be protective and not detrimental dietary compounds, and did not include alcohol consumption in the index ${ }^{(28)}$. The total MD score ranged from 0 (minimal adherence to the traditional MD) to 8 (maximal adherence). The score was categorised to reflect three levels of adherence: (1) $\leq 3$, low; (2) 4-5, medium; (3) 6-8, high MD quality.

\section{Fetal growth assessment}

Information on anthropometric measures at birth was obtained from the hospital delivery logs and medical records in both cohorts. Gestational age was based on the interval between the last menstrual period and the date of delivery for $84 \%$ of the participants in RHEA cohort and $88 \%$ of the participants in INMA cohort. When the menstrual estimate of gestational age was inconsistent by 7 or more days with the ultrasound measurement taken in the first trimester of pregnancy (RHEA cohort: 16\%; INMA cohort: 12\%), a quadratic regression formula describing the relationship between crown-rump length and gestational age was used instead ${ }^{(29)}$.

Fetal growth restriction after accounting for growth potential. A customised definition of restricted growth for the newborns of this study was developed taking into account their constitutional characteristics ${ }^{(30,31)}$. Maternal and newborn characteristics considered a priori were: gestational age in weeks, maternal and paternal height (in $\mathrm{cm}$ ) and age (in years), maternal pre-pregnancy weight (in $\mathrm{kg}$ ), primiparous mother and infant sex. Gestational age and infant sex were considered as possible modifiers. A multivariable fractional polynomial linear regression model was used to predict BW, allowing polynomial terms for continuous variables in the linear regression models ${ }^{(31)}$. The model with the minimum deviance (i.e. best fit) was retained as the final model, and included as covariates for the RHEA cohort: gestational age, infant sex, maternal and paternal height, pre-pregnancy weight as well as the interaction of gestational age with maternal weight; and for the INMA cohort: gestational age, infant sex, maternal and paternal height, pre-pregnancy weight and parity.

A neonate was defined as having fetal weight growth restriction (FwGR) if his/her actual BW fell below the 10th percentile of the predicted BW distribution. Similar models were used to define neonates with fetal length growth restriction (FlGR) if his/her actual birth length (BL) fell below the 10th percentile of the predicted $\mathrm{BW}$ distribution, and to define neonates with fetal head circumference growth restriction (FhcGR) if his/her actual BL fell below the 10th percentile of the predicted birth head circumference (HC).

\section{Potential confounders}

Potential confounders included characteristics that have an established or potential association with preterm birth, fetal 
growth restriction and MD in pregnancy including: maternal and paternal age; maternal and paternal education (low level: $\leq 6$ years of school, medium level: $\leq 12$ years of school, high level: university of technical college degree); maternal and paternal social class defined in three occupational categories according to their present or most recent occupation, following a widely used Spanish adaptation of the British classification system (I + II, III, IV + V) $)^{(32)}$; maternal pre-pregnancy weight $(\mathrm{kg})$, maternal pre-pregnancy and paternal BMI $\left(\mathrm{kg} / \mathrm{m}^{2}\right)$; weight gain during pregnancy $(\mathrm{kg})$, maternal smoking during pregnancy (yes/no); parity (primiparous, multiparous); infant sex (male, female), use of multivitamins containing $\mathrm{Ca}$, Fe and folic acid during pregnancy (first trimester for INMA cohort, and mid pregnancy for RHEA cohort), alcohol intake during pregnancy ( $\mathrm{g} / \mathrm{d}$ for the first trimester in INMA cohort, and for mid-pregnancy for RHEA cohort), gestational hypertension and pre-eclampsia, diabetes before pregnancy and total energy intake during pregnancy $(\mathrm{kJ} / \mathrm{d}$ for the first trimester in INMA cohort, and for mid-pregnancy for RHEA cohort).

\section{Statistical analysis}

Data analysis was performed using STATA version 10.0 (StataCorp LP, College Station, TX, USA). The primary outcome variables of interest were $\mathrm{BW}, \mathrm{BL}, \mathrm{HC}$ and fetal growth restriction. The primary exposure of interest was the adherence to the MD during pregnancy. Bivariate associations between dependent and independent variables were studied using Pearson's $\chi^{2}$ test for categorical variables or KruskalWallis test for continuous ones.

Multivariable log-binomial and linear regression models were further performed to examine the association between MD in pregnancy and the outcomes of interest after adjusting for confounders. Potential confounders related with the outcomes of interest in the bivariate models with a $P$ value $<0 \cdot 2$ were included in the multivariable models. All analyses were adjusted for total energy intake using the residual method $^{(33)}$. Relative risks with $95 \% \mathrm{CI}$, and coefficients $(\beta)$ with SE were computed to estimate the degree of association. To account for the possibility of residual confounding, the remaining demographic, lifestyle and pregnancy characteristics that were available in this data set were then sequentially forced into the parsimonious models to ensure that the estimates associated with MD remained unchanged. Effect modification was evaluated using the likelihood ratio test and stratified models were obtained. Adjusted means for each stratum were also derived for continuous outcomes from linear combinations of regression coefficients. All association testing was conducted assuming a 0.05 significance level and a two-sided alternative hypothesis.

\section{Results}

The distribution of baseline characteristics of women participating in the Spanish cohorts in the Atlantic area (INMAAtlantic), the Mediterranean area (INMA-Mediterranean) and the Cretan cohort (RHEA) according to MD score is shown in Table 1. Women with a high adherence to MD during pregnancy in INMA-Mediterranean were more likely to be older and non-smokers, women in INMA-Atlantic were more likely to be primiparous, while in the RHEA cohort, women were more likely to be older and highly educated. In all cohorts women with a high adherence to MD had higher total energy intake during pregnancy compared with those with a medium or low adherence to MD.

Food group intakes differed significantly across cohorts (Figs. 1 and 2). Women in INMA-Atlantic reported higher intakes of fish (median $71.5 \mathrm{~g}$ ), legumes (median 60.2 g) and dairy products (median $548.7 \mathrm{~g}$ ); women in INMAMediterranean reported higher intakes of cereals (median $161.3 \mathrm{~g}$ ) while women in RHEA had higher intakes of fruits and nuts (median $403.1 \mathrm{~g}$ ). Fig. 3 shows the distribution of MD scores in the INMA and RHEA cohorts. In the INMA-Atlantic cohort there was the highest proportion of subjects with low adherence to the MD (49.9\%), while the RHEA cohort had the highest proportion of subjects with high adherence (18.5\%). Similarly, the mean MD score was higher in the Mediterranean cohorts (INMA Mediterranean: $3 \cdot 8$ (SD 1.6); RHEA cohort: 4.0 (SD 1.7)) compared with the Atlantic cohorts (INMA Atlantic: mean 3.5, SD 1.4).

In INMA-Atlantic, mean BW, BL and $\mathrm{HC}$ values were $3283 \cdot 2$ (SD 465) g, $49 \cdot 3(\mathrm{SD} 2 \cdot 1) \mathrm{cm}$ and 34.5 (SD 1.4 ) cm, respectively. The prevalence of FwGR, FlGR and FhcGR was 8.9, 9.1 and $9.6 \%$, respectively. In INMA-Mediterranean, mean $\mathrm{BW}, \mathrm{BL}$ and $\mathrm{HC}$ values were 3233.2 (SD 188) g, 49.7 (SD 2.4) cm and 34.1 (SD 1.5$) \mathrm{cm}$, respectively. The prevalence of FwGR, FlGR and FhcGR was $10.3,9 \cdot 2$ and $9.9 \%$, respectively. In the RHEA cohort, mean BW, BL and HC values were 3206 (SD 462) g, $50.5(\mathrm{SD} 0.5) \mathrm{cm}$ and $34.2(\mathrm{SD} 1.5) \mathrm{cm}$, respectively. The prevalence of FwGR, FlGR and FhcGR was 8.0, 6.7 and $8 \cdot 3 \%$, respectively.

Tables 2 and 3 present the associations between anthropometric measurements at birth and fetal growth restriction, respectively, with MD scores during pregnancy in the INMA and RHEA cohorts after adjusting for several confounders. In the INMA-Mediterranean cohort, high MD adherence was associated with a BW gain of 88 (SE 33.4) $\mathrm{g}$ and length gain of $0.30(\mathrm{se} 0 \cdot 1) \mathrm{cm}$, while in the INMA-Atlantic and RHEA cohorts, a high adherence to the MD was inversely associated with anthropometric measurements at birth, though associations were not statistically significant. Women with high MD adherence had a significantly lower risk of delivering a fetal growth-restricted infant for weight (relative risks: 0.5; $95 \%$ CI $0.3,0.9$ ) in INMA-Mediterranean, but this was not the case for the INMA-Atlantic and RHEA cohorts.

Fig. 4 presents the stratified analysis by smoking status for the associations between anthropometric measurements at birth with MD scores during pregnancy in the INMA and RHEA cohorts after adjusting for several confounders. In smoking mothers from the INMA-Atlantic cohort, a higher MD adherence increased BW by 319 (sE 124.3) g and BL by $1.3(\mathrm{se} 0.6) \mathrm{cm}$, while the effect was negative for nonsmoking mothers ( $P$ value for interaction 0.016 and 0.010, respectively). Similarly in the INMA Mediterranean cohort, smoking mothers with high adherence to MD during 
Table 1. Maternal socio-demographic and lifestyle factors by Mediterranean diet (MD) score in pregnancy in the Spanish INMA and the Greek RHEA mother and child cohort studies

\begin{tabular}{|c|c|c|c|c|c|c|c|c|c|c|c|c|}
\hline & \multicolumn{4}{|c|}{ INMA-Atlantic } & \multicolumn{4}{|c|}{ INMA-Mediterranean } & \multicolumn{4}{|c|}{ RHEA } \\
\hline & \multicolumn{4}{|c|}{ MD score* } & \multicolumn{4}{|c|}{ MD score } & \multicolumn{4}{|c|}{ MD score } \\
\hline & $\begin{array}{l}\text { Low } \\
(n 535)\end{array}$ & $\begin{array}{l}\text { Medium } \\
(n \text { 453) }\end{array}$ & $\begin{array}{l}\text { High } \\
(n \text { 86) }\end{array}$ & $P \dagger$ & $\begin{array}{c}\text { Low } \\
(n 583)\end{array}$ & $\begin{array}{c}\text { Medium } \\
\text { (n 593) }\end{array}$ & $\begin{array}{l}\text { High } \\
(n 210)\end{array}$ & $P \dagger$ & $\begin{array}{l}\text { Low } \\
(n 327)\end{array}$ & $\begin{array}{l}\text { Medium } \\
(n \text { 325) }\end{array}$ & $\begin{array}{l}\text { High } \\
(n \text { 172) }\end{array}$ & $P \dagger$ \\
\hline Maternal age (years) $\ddagger$ & $31 \cdot 2$ & 31.6 & $31 \cdot 7$ & 0.238 & 29.4 & 30.3 & $30 \cdot 8$ & $<0.001$ & $28 \cdot 8$ & 30.0 & $29 \cdot 7$ & 0.006 \\
\hline Paternal age (years) $\ddagger$ & 33.5 & 33.9 & 34.5 & 0.212 & 31.4 & 32.4 & $32 \cdot 8$ & $<0.001$ & 33.2 & $34 \cdot 2$ & $33 \cdot 7$ & 0.073 \\
\hline Maternal pre-pregnancy BMI $\left(\mathrm{kg} / \mathrm{m}^{2}\right) \ddagger$ & 23.2 & 23.4 & 23.8 & 0.371 & 23.9 & 23.7 & 23.4 & 0.510 & $24 \cdot 4$ & 24.1 & $24 \cdot 3$ & 0.756 \\
\hline Paternal BMI $\left(\mathrm{kg} / \mathrm{m}^{2}\right) \ddagger$ & $26 \cdot 0$ & $26 \cdot 0$ & $25 \cdot 9$ & 0.996 & $25 \cdot 9$ & $26 \cdot 0$ & $25 \cdot 3$ & 0.059 & $27 \cdot 6$ & $27 \cdot 1$ & $27 \cdot 9$ & 0.302 \\
\hline Alcohol intake during pregnancy $(\mathrm{g} / \mathrm{d}) \ddagger$ & $3 \cdot 3$ & $2 \cdot 0$ & 1.0 & 0.642 & $5 \cdot 8$ & 4.2 & $6 \cdot 1$ & 0.725 & $7 \cdot 8$ & 13.5 & 8.1 & 0.597 \\
\hline Energy intake during pregnancy $(\mathrm{kJ} / \mathrm{d}) \ddagger$ & $7752 \cdot 1$ & 8873.4 & 9734.5 & $<0.001$ & $8612 \cdot 8$ & $9476 \cdot 8$ & $10398 \cdot 1$ & $<0.001$ & $7501 \cdot 1$ & $8861 \cdot 7$ & $10306 \cdot 9$ & $<0.001$ \\
\hline Gestational age (weeks) $\ddagger$ & $39 \cdot 6$ & 39.6 & $39 \cdot 6$ & 0.871 & 39.7 & 39.5 & 39.5 & 0.259 & 38.3 & 38.3 & $38 \cdot 1$ & 0.476 \\
\hline \multicolumn{13}{|l|}{ Parity (\%) } \\
\hline Primiparous & 48.4 & 44.2 & $52 \cdot 3$ & & 44.3 & 44.5 & $45 \cdot 7$ & & $35 \cdot 8$ & $36 \cdot 2$ & $43 \cdot 2$ & \\
\hline Multiparous & $51 \cdot 6$ & 55.8 & $47 \cdot 7$ & 0.020 & $55 \cdot 7$ & 55.5 & 54.3 & 0.071 & $64 \cdot 2$ & $63 \cdot 1$ & $56 \cdot 8$ & 0.193 \\
\hline \multicolumn{13}{|l|}{ Maternal education (\%) } \\
\hline Primary or less & $15 \cdot 0$ & $15 \cdot 7$ & 17.4 & & $30 \cdot 2$ & 32.6 & 28.6 & & $26 \cdot 1$ & $17 \cdot 6$ & 11.6 & \\
\hline Secondary & $40 \cdot 9$ & 38.0 & $36 \cdot 0$ & & 41.8 & $42 \cdot 2$ & $47 \cdot 1$ & & $46 \cdot 9$ & 51.4 & $55 \cdot 2$ & \\
\hline University & $44 \cdot 1$ & $46 \cdot 4$ & $46 \cdot 5$ & 0.845 & $28 \cdot 0$ & $25 \cdot 2$ & $24 \cdot 3$ & 0.497 & $27 \cdot 0$ & 31.0 & $33 \cdot 1$ & 0.003 \\
\hline \multicolumn{13}{|l|}{ Paternal education (\%) } \\
\hline Primary or less & $28 \cdot 0$ & $26 \cdot 7$ & 23.5 & & 41.9 & $43 \cdot 2$ & $36 \cdot 4$ & & 39.7 & $33 \cdot 2$ & 34.7 & \\
\hline Secondary & $47 \cdot 3$ & $48 \cdot 2$ & $52 \cdot 9$ & & $40 \cdot 8$ & 39.8 & 43.5 & & 40.9 & $41 \cdot 7$ & 44.7 & \\
\hline University & $24 \cdot 8$ & $25 \cdot 1$ & 23.5 & 0.887 & $17 \cdot 3$ & $16 \cdot 9$ & $20 \cdot 1$ & 0.526 & $19 \cdot 4$ & $25 \cdot 1$ & $20 \cdot 6$ & 0.2 \\
\hline \multicolumn{13}{|l|}{ Maternal social class (\%)§ } \\
\hline I-II & $25 \cdot 6$ & 27.8 & $30 \cdot 6$ & & $18 \cdot 7$ & $15 \cdot 3$ & $21 \cdot 0$ & & - & - & - & \\
\hline III & $26 \cdot 2$ & 23.8 & $21 \cdot 2$ & & $26 \cdot 8$ & 26.5 & $27 \cdot 6$ & & - & - & - & \\
\hline IV & 48.2 & $48 \cdot 3$ & 48.2 & 0.750 & 54.5 & $58 \cdot 2$ & $51 \cdot 4$ & 0.286 & - & _- & - & \\
\hline \multicolumn{13}{|l|}{ Paternal social class (\%)§ } \\
\hline I-II & $22 \cdot 6$ & 23.5 & $25 \cdot 6$ & & $18 \cdot 0$ & $15 \cdot 4$ & $18 \cdot 6$ & & - & - & - & \\
\hline III & $15 \cdot 0$ & $16 \cdot 7$ & $12 \cdot 2$ & & $19 \cdot 0$ & $18 \cdot 0$ & $21 \cdot 1$ & & - & _- & - & \\
\hline IV & $62 \cdot 4$ & 59.8 & $62 \cdot 2$ & 0.801 & $63 \cdot 0$ & 66.5 & $60 \cdot 3$ & 0.502 & - & - & - & \\
\hline \multicolumn{13}{|l|}{ Smoking during pregnancy (\%) } \\
\hline No & $87 \cdot 2$ & 83.2 & 81.9 & & $76 \cdot 7$ & $81 \cdot 0$ & 83.9 & & $76 \cdot 2$ & 77.5 & $77 \cdot 3$ & \\
\hline Yes & $12 \cdot \overline{8}$ & $16 \cdot 8$ & $18 \cdot 1$ & 0.162 & $23 \cdot 3$ & $19 \cdot 0$ & $16 \cdot 1$ & 0.049 & 23.8 & 22.5 & 22.7 & 0.916 \\
\hline \multicolumn{13}{|l|}{ Gestational hypertension (\%) } \\
\hline No & $98 \cdot 3$ & $97 \cdot 8$ & $97 \cdot 7$ & & $97 \cdot 1$ & 97.6 & $98 \cdot 1$ & & $96 \cdot 8$ & 96.4 & $97 \cdot 4$ & \\
\hline Yes & 1.7 & $2 \cdot 2$ & $2 \cdot 3$ & 0.741 & 2.9 & 2.4 & 1.9 & 0.736 & 3.2 & 3.6 & 2.6 & 0.903 \\
\hline \multicolumn{13}{|l|}{ Diabetes before pregnancy (\%) } \\
\hline No & 99.8 & 99.6 & $100 \cdot 0$ & & 99.7 & 99.8 & 99.5 & & 97.7 & 98.0 & 98.0 & \\
\hline Yes & 0.2 & 0.4 & 0.0 & 0.685 & 0.3 & 0.2 & 0.5 & 0.665 & $2 \cdot 3$ & 2.0 & 2.0 & 0.9 \\
\hline \multicolumn{13}{|l|}{ Infant sex (\%) } \\
\hline Female & $50 \cdot 5$ & 48.7 & $36 \cdot 0$ & & 50.9 & 46.0 & 46.9 & & $45 \cdot 0$ & 51.4 & $47 \cdot 7$ & \\
\hline Male & 49.5 & 51.3 & 64.0 & 0.046 & $49 \cdot 1$ & 54.0 & $53 \cdot 1$ & 0.221 & 55.0 & 48.6 & $52 \cdot 3$ & 0.258 \\
\hline
\end{tabular}

"Mediterranean diet score: low, 0-3; medium, 4-5; high, 6-8.

$\dagger$ values obtained by using Pearson's $\chi^{2}$ test for categorical variables or Student's $t$ test/ANOVA for continuous ones.

$\mp$ Values are expressed as means.

$\S$ Social classes: I-II, professional-managerial; III, skilled (manual and non-manual); IV, partly skilled and unskilled, housewife. 


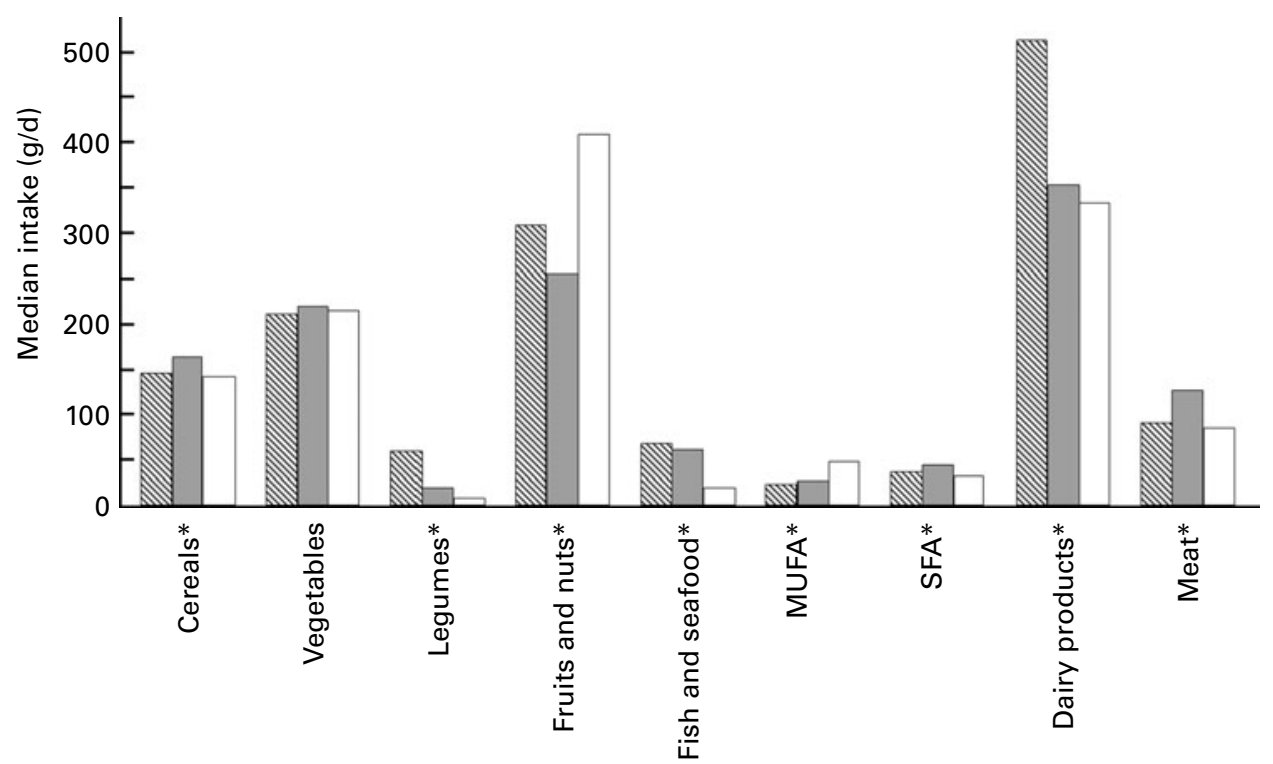

Fig. 1. Food group intake during pregnancy in INMA and RHEA mother-child cohort studies. In INMA cohort the dietary intake refers to the first trimester of pregnancy; in RHEA cohort dietary intake refers to the first $4-5$ months of pregnancy. ${ }^{*}$ Values were significantly different $(P<0.001)$. $\mathbb{\mathbb { S }}$, INMA Atlantic; $\square$, INMA Mediterranean; $\square$, RHEA.

pregnancy had an increase by 200 (SE 81.5) $\mathrm{g}$ in BW and by $0.8(\mathrm{SE} 0.4) \mathrm{cm}$ in $\mathrm{BL}$, whereas there was no such effect in non-smoking mothers ( $P$ value for interaction 0.272 and 0.062 , respectively). A similar pattern has been shown for smokers and non-smokers in the RHEA cohort, though the associations were not statistically significant.

Separate analysis for each food group included in the MD score (by SD of daily intake in $\mathrm{g}$ ) has shown that an increase by $47 \mathrm{~g}$ in fish and seafood intake was associated with a reduction of $\mathrm{BL}$ by $1.3(\mathrm{SE} 0.06) \mathrm{cm}$ in the INMA Atlantic cohort, an increase by $126 \mathrm{~g}$ in vegetable intake was associated with a BW gain of 28.0 (SE 11.3) g in the INMA-Mediterranean cohort, while an increase by 0.22 in the ratio of monounsaturated lipids to saturated lipids was associated with a BW gain

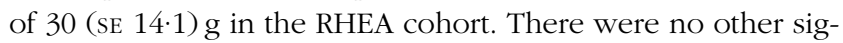
nificant associations between food groups' intake and reproductive outcomes in the three cohorts.

To assess whether disparities in fish consumption patterns in the INMA-Atlantic cohort might explain the smoking-MD adherence interaction observed in that region but not in the other cohorts, we compared intakes of different types of fish across regions. Women in the INMA-Atlantic cohort

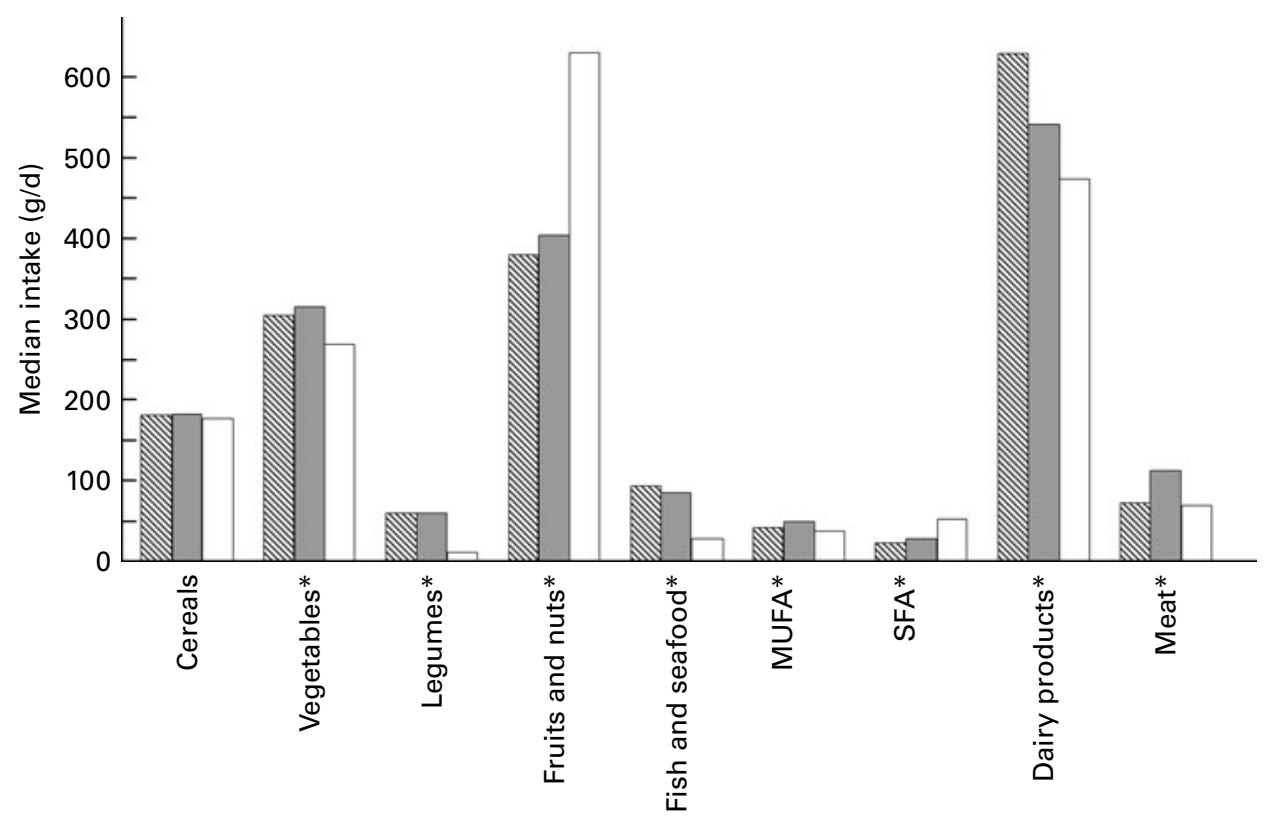

Fig. 2. Food group intake among women with the highest adherence to the Mediterranean diet during pregnancy in INMA and RHEA mother-child cohort studies. In INMA cohort the dietary intake refers to the first trimester of pregnancy; in RHEA cohort dietary intake refers to the first $4-5$ months of pregnancy. ${ }^{*}$ Values

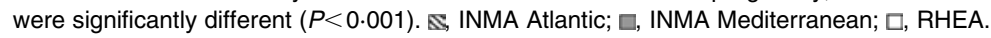




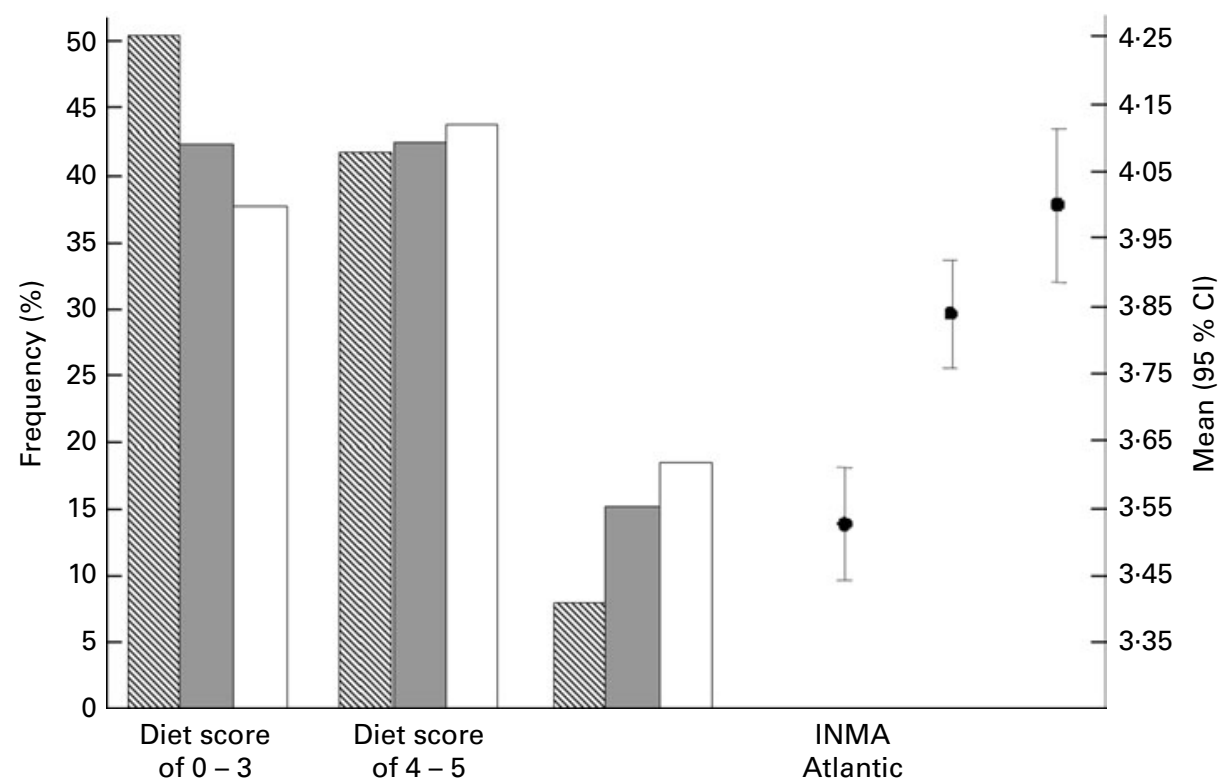

Fig. 3. Mediterranean diet score during pregnancy in INMA and RHEA mother-child cohort studies. In INMA cohort the dietary intake refers to the first trimester of pregnancy; in RHEA cohort dietary intake refers to the first 4-5 months of pregnancy. $\mathbb{\$}$, INMA Atlantic; $\square$, INMA Mediterranean; $\square$, RHEA.

had the highest fish and seafood intake during pregnancy (77.2 (SD 47.2) g) compared with the other cohorts (INMAMediterranean: 66.1 (SD 36.4) g; RHEA: 24.2 (SD 27.4) g, $P<0 \cdot 001)$. To evaluate further the possibility of effect modification by smoking regarding fish consumption and fetal growth in the INMA-Atlantic cohort, we included the variable for fish intake, the variable for smoking status during pregnancy and a term for interaction between the two variables, both in univariate and multivariate models (data not shown). In all the cases, the interaction term was nonsignificant, although fish intake in smoking mothers was associated with increase in BW, while in non-smoking mothers it was associated with decrease in BW.

To evaluate the possibility of confounding by gestational hypertension, diabetes before pregnancy and preterm birth, we carried out additional analyses excluding pregnancies complicated by gestational hypertension (INMA cohort: $n 57$ (2.3\%), RHEA cohort: $n 30(3 \cdot 4 \%)$ ), diabetes before pregnancy (INMA cohort: $n 23$ (0.9\%), RHEA cohort: $n 20$ $(2 \cdot 2 \%)$ ) and preterm births (INMA cohort: $n 113(4.6 \%)$, RHEA cohort: $n 98(11 \cdot 2 \%)$ ), and the results remained essentially the same with the original analysis (data not shown).

Table 2. Associations between Mediterranean diet (MD) score* in pregnancy with anthropometric measurements at birth in INMA and RHEA motherchild cohort studies

( $\beta$ Coefficients and standard errors)

\begin{tabular}{|c|c|c|c|c|c|c|c|c|c|}
\hline \multirow[b]{2}{*}{ MD score } & \multicolumn{3}{|c|}{ INMA-Atlantic } & \multicolumn{3}{|c|}{ INMA-Mediterranean } & \multicolumn{3}{|c|}{ RHEA } \\
\hline & $\beta$ & SE & $P \dagger$ & $\beta$ & SE & $P+$ & $\beta$ & SE & $P \dagger$ \\
\hline \multicolumn{10}{|l|}{ Weight‡ } \\
\hline Low & Ref. & & & Ref. & & & Ref. & & \\
\hline Medium & $-26 \cdot 46$ & 26.03 & 0.310 & $55 \cdot 20$ & 23.52 & 0.019 & -33.67 & $31 \cdot 78$ & 0.290 \\
\hline High & $-82 \cdot 85$ & $47 \cdot 67$ & 0.082 & $87 \cdot 78$ & 33.40 & 0.009 & $-20 \cdot 42$ & $42 \cdot 33$ & 0.630 \\
\hline \multicolumn{10}{|l|}{ Length§ } \\
\hline Low & Ref. & & & Ref. & & & Ref. & & \\
\hline Medium & -0.16 & 0.12 & 0.185 & 0.13 & $0 \cdot 10$ & 0.203 & -0.43 & $0 \cdot 18$ & 0.077 \\
\hline High & -0.25 & 0.22 & 0.245 & 0.30 & 0.15 & 0.040 & -0.06 & 0.24 & 0.794 \\
\hline \multicolumn{10}{|c|}{ Head circumferencell } \\
\hline Low & Ref. & & & Ref. & & & Ref. & & \\
\hline Medium & 0.03 & 0.09 & 0.770 & 0.03 & 0.07 & 0.647 & -0.23 & $0 \cdot 12$ & 0.049 \\
\hline High & -0.06 & $0 \cdot 16$ & 0.711 & $0 \cdot 16$ & $0 \cdot 10$ & 0.121 & -0.20 & $0 \cdot 16$ & 0.214 \\
\hline
\end{tabular}

Ref., reference category.

* Mediterranean diet score: low, 0-3; medium, 4-5; high, 6-8. All models were adjusted for sex, gestational age, maternal smoking during pregnancy, maternal age and total energy intake using linear regression models.

$\dagger P$ values of each component from the regression model.

¥ Also adjusted for: INMA-Atlantic: parity, maternal BMI, paternal education and parental social class; INMA-Mediterranean: parity, parental BMI, and maternal social class; RHEA: maternal BMI and education.

$\S$ Also adjusted for: INMA-Atlantic: parity, maternal BMI, paternal age and maternal social class; INMA-Mediterranean: parity, maternal BMI and maternal social class; RHEA: maternal height and education.

II Also adjusted for: INMA-Atlantic: parity, maternal BMI and education; INMA-Mediterranean: parity, maternal BMI, maternal education and alcohol intake; RHEA: maternal BMI and education. 
Table 3. Associations between Mediterranean diet (MD) score* in pregnancy with fetal growth restriction in INMA and RHEA mother-child cohort studies

(Relative risks (RR) and 95\% confidence intervals)

\begin{tabular}{|c|c|c|c|c|c|c|c|c|c|}
\hline \multirow[b]{2}{*}{ MD score } & \multicolumn{3}{|c|}{ INMA-Atlantic } & \multicolumn{3}{|c|}{ INMA-Mediterranean } & \multicolumn{3}{|c|}{ RHEA } \\
\hline & $\mathrm{RR}$ & $95 \% \mathrm{Cl}$ & $P+$ & $\mathrm{RR}$ & $95 \% \mathrm{Cl}$ & $P+$ & $\mathrm{RR}$ & $95 \% \mathrm{Cl}$ & $P+$ \\
\hline \multicolumn{10}{|c|}{ FGR in weight $\ddagger$} \\
\hline Low & Ref. & & & Ref. & & & Ref. & & \\
\hline Medium & $1 \cdot 24$ & $0.81,1.89$ & 0.329 & 0.76 & $0.54,1.06$ & 0.109 & 1.82 & $0.95,3.49$ & 0.071 \\
\hline High & 0.97 & $0.42,2.26$ & 0.943 & 0.50 & $0.28,0.90$ & 0.021 & 1.96 & $0.90,4.25$ & 0.089 \\
\hline \multicolumn{10}{|c|}{ FGR in length§ } \\
\hline Low & Ref. & & & Ref. & & & Ref. & & \\
\hline Medium & 1.33 & $0.87,2.04$ & 0.189 & 1.01 & $0.70,1.47$ & 0.952 & 1.39 & $0.72,2.68$ & 0.330 \\
\hline High & 0.63 & $0.23,1.76$ & 0.376 & 0.95 & $0.55,1.62$ & 0.838 & 0.90 & $0.35,2.30$ & 0.821 \\
\hline \multicolumn{10}{|c|}{ FGR in head circumference\| } \\
\hline Low & Ref. & & & Ref. & & & Ref. & & \\
\hline Medium & 0.88 & $0.57,1.34$ & 0.543 & $1 \cdot 15$ & $0.80,1.65$ & 0.459 & 1.63 & $0.89,2.96$ & $0 \cdot 112$ \\
\hline High & $1 \cdot 11$ & $0.53,2.33$ & 0.779 & 1.07 & $0.63,1.83$ & 0.800 & 1.64 & $0.76,3.56$ & 0.211 \\
\hline
\end{tabular}

Ref., reference category.

* Mediterranean diet score: low, 0-3; medium, 4-5; high, 6-8. All models were adjusted for maternal smoking during pregnancy, maternal age and total energy intake. $\dagger P$ values for each component from the log-binomial model.

Also adjusted for: INMA-Atlantic: maternal social class; INMA-Mediterranean: maternal BMI and maternal social class; RHEA: paternal age and maternal education.

$\S$ Also adjusted for: INMA-Atlantic and INMA-Mediterranean: maternal education; RHEA: paternal education.

I| Also adjusted for: INMA-Atlantic: paternal age, maternal BMI and education; INMA-Mediterranean: maternal BMI, alcohol intake, education and social class; RHEA: maternal alcohol intake and education.

\section{Discussion}

We evaluated MD adherence during pregnancy in two large population-based mother and child cohorts in Spain and Greece in association with birth outcomes. MD scores differ significantly between the Spanish cohorts in the Atlantic and the Mediterranean areas, and the Cretan cohort. Women with high MD adherence had a significantly lower risk of delivering a fetal growth-restricted infant for weight in INMAMediterranean, but this was not the case for the INMA-Atlantic or RHEA cohort. Stratified analysis by smoking revealed that higher MD adherence increased BW and BL in smoking mothers, whereas this effect was not apparent in non-smoking mothers. To the best of our knowledge, this is the first study to assess prospectively the impact of MD during pregnancy on anthropometric measurements at birth in general populations across the Mediterranean.
Research on child health effects of maternal nutrition has focused largely on deficiencies, and there are very little data available describing prevailing patterns of dietary intake among pregnant women in developed countries, including levels of compliance with dietary guidelines, or degree of adherence to potentially healthful dietary patterns such as the MD. The protective effect of a high adherence to MD in fetal growth in the INMA-Mediterranean cohort is in accordance with previous analyses within the INMA cohort in Valencia that have shown a significant increase in BW and BL in newborns of women with better diet during the first trimester based on the Alternate Healthy Eating Index ${ }^{(20)}$, and a positive association of vegetable intake during pregnancy with anthropometric measurements at birth $^{(7)}$.

Dietary patterns account for cumulative and interactive effects among nutrients, reflect real-world dietary preferences and may be particularly suitable for analysis in epidemiology
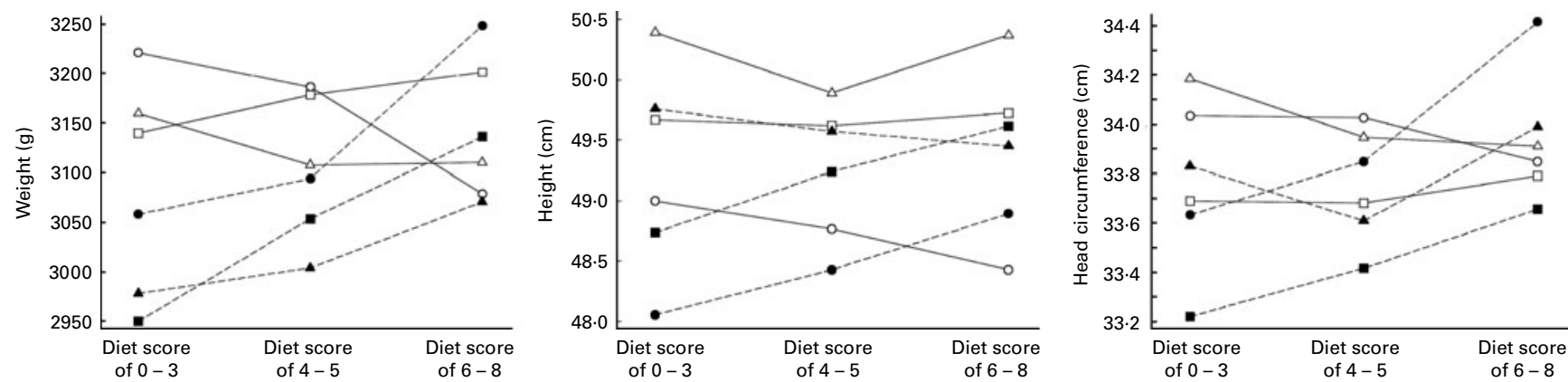

Fig. 4. Adjusted means of anthropometric measurements at birth by Mediterranean diet score and maternal smoking during pregnancy in INMA and RHEA mother and child cohort studies. Results are shown for INMA-Atlantic: non-smokers (O), smokers (•); INMA-Mediterranean: non-smokers ( $\square$ ), smokers ( $\square$ ); RHEA: nonsmokers $(\triangle)$, smokers $(\boldsymbol{\Lambda})$. All models were adjusted for maternal age and total energy intake, sex and gestational age. Models for weight were also adjusted for: INMA-Atlantic: parity, maternal BMI, paternal education and parental social class; INMA-Mediterranean: parity, parental BMI and maternal social class; RHEA: maternal BMI and education. Models for height were also adjusted for: INMA-Atlantic: parity, maternal BMI, paternal age and maternal social class; INMA-Mediterranean: parity, maternal BMI and maternal social class; RHEA: maternal height and education. Models for head circumference were also adjusted: INMA-Atlantic: parity, maternal BMI and education; INMA-Mediterranean: parity, maternal BMI, maternal education and alcohol intake; RHEA: maternal BMI and education. 
of fetal growth in which many dietary components could be related with the outcome of interest ${ }^{(34)}$. The traditional MD refers to the dietary pattern in the Mediterranean olive grove areas at the beginning of the $1960 \mathrm{~s}^{(35)}$. However, the MD presents regional variations derived from the same dietary pattern, influenced by various factors, such as sociocultural, religious and economic determinants ${ }^{(36)}$. As expected, in the present study, MD during pregnancy was not homogeneous, with women in the Atlantic area reporting higher intakes of fish and dairy products, while women in Crete reported higher intakes of fruits and nuts. This could explain in part the different results in the present study across the different regions. For example, the negative associations with BW and BL that were found in non-smoking women with the highest adherence to the MD in the INMA-Atlantic cohort might reflect the high consumption of fish and seafood in this population, a known route of exposure to persistent organic pollutants as confirmed in previous cohort studies ${ }^{(7,10,12)}$. Stratified analysis by smoking in the INMA-Atlantic cohort has shown that although fish intake in smoking mothers was associated with increase in BW, while with decrease in non-smoking mothers, the associations were not statistical significant, therefore the underlying pathophysiology of this association remains unclear.

Cigarette smoking during pregnancy has been previously associated with low BW in a number of studies ${ }^{(37-40)}$, and has been causally indicated to cause a reduction of 100$300 \mathrm{~g}$, while other research has indicated even an about $500 \mathrm{~g}$ reduction among populations with certain metabolic gene polymorphisms ${ }^{(41)}$. The results of the present study indicate that a high adherence to MD during pregnancy in smoking women could increase $\mathrm{BW}$ and $\mathrm{BL}$, and decrease the risk for fetal growth retardation. Numerous studies have shown that smokers generally have poorer-quality diets than nonsmokers, with lower levels of antioxidant-rich foods such as fruits and vegetables ${ }^{(42)}$. Therefore, the protective effect of maternal adherence to MD during pregnancy in smokers might reflect high fetal exposure to several antioxidant compounds, and their property to counter the effect of oxidative stress damage of fetal tissues.

Adherence to the MD during pregnancy was evaluated according to an index widely used for the evaluation of the adherence to the MD in adults ${ }^{(27)}$. In the present study, we evaluated maternal adherence to the MD in pregnancy in relation to several birth outcomes. Consequently, some questions could arise regarding the effectiveness of the MD in pregnancy in relation to the increased maternal nutritional needs during this period. First, several studies have demonstrated the high nutritional quality of the MD characterised by better dietary fat quality and an increased quantity of antioxidants $^{(43,44)}$. Because alcohol consumption is prohibited in pregnancy, and during this period there is increased need for $\mathrm{Ca}$ intake, we did not include in the index alcohol consumption and we presumed dairy compounds to be protective and not detrimental. Moreover, clinical outcome studies suggest that bone mineral mass in newborn infants is related to maternal size and dairy product intake during pregnancy $^{(45)}$.
The strengths of the present study include the populationbased multi-centre prospective design, the large numbers of children with anthropometric measurements at birth and the detailed data for dietary intake during pregnancy. The study used a novel term, 'fetal growth restriction', to define an infant who has not achieved its genetic growth potential in the uterus on the basis of customised BW, BL or HC CI. This model allowed us to classify neonates as fetal growth restricted, and to differentiate them from those being only constitutionally small ${ }^{(23,31)}$. The study population included women from the follow-up of several birth cohorts, giving us the opportunity to account for the effect of exposures assessed prospectively within the cohorts. As the INMA and RHEA cohorts used different methodology for the estimation of nutrient intakes, we decided not to pool data from the two cohorts but to assess the impact of MD in each cohort separately. We did not observe any substantial differences between the crude and the adjusted models. Thus, it is unlikely that over-adjustment affected the findings of the present study. Participants were unaware of the MD hypothesis being tested, so, misclassification of intake estimated by the FFQ is likely to be random with respect to birth outcomes. Unlike previous epidemiological studies, the adherence to the MD during pregnancy was adjusted for total energy intake, which allowed for different individual requirements. To evaluate the possibility of introducing confounding by supplement intake and weight gain during pregnancy, we performed additional analyses including these variables as possible confounders in the multivariate models, and the results remained essentially the same with the original analysis (data not shown).

There are several limitations in the present study that deserve acknowledgement. As in most studies on diet and health, we used self-reported diet during pregnancy, and therefore, information bias could have occurred. However, studies of nutrition in pregnancy suggest that food frequency methods could present valid and reproducible estimates of the dietary intakes in pregnant women ${ }^{(46)}$. Indexes like MD score that utilises scorings have certain limitations such as the variability in choosing cut-off points in the score and the different distribution of selected food groups in different populations ${ }^{(36)}$. A sensitivity analysis using MD score in pregnancy as a continuous variable gave essentially the same results with the categorical analysis (data not shown). Unfortunately, information on gestational diabetes was not available for some INMA sub-cohorts and we did not have information on dietary intervention for treating gestational diabetes; therefore, we could not explore the association of adherence to the MD during pregnancy on gestational diabetes and its consequences on birth outcomes. Although we incorporated extensive information on potential social and environmental factors that are associated with fetal growth, we acknowledge that there may be other factors linked with both fetal growth and MD during pregnancy that could explain this association.

In summary, these results suggest that adherence to MD during pregnancy is not homogeneous within the Mediterranean area. A high adherence to MD may modify the detrimental effect of smoking on birth size, but overall effects of diet 
were not universal for the studies in this analysis. The complex underlying processes that explain these findings require additional study. Further follow-up of these cohorts will allow determining if a higher adherence to MD during pregnancy has, in addition, an effect on growth and neurodevelopment in childhood.

\section{Acknowledgements}

All the authors had a substantial contribution to the study and they have all personally reviewed and approved the submitted manuscript. L. C. conceived the study, conducted the data analysis and prepared the manuscript; M. M. contributed to the design of the study and the drafting of the manuscript; R. G. and T. R. carried out the statistical and helped with the data interpretation and manuscript preparation; J. I., A. T., P. A. and A. L. supervised the data collection and helped with manuscript preparation; C. I. developed the model for fetal growth restriction and assisted with manuscript preparation; J. V. designed the FFQ for INMA cohort and contributed to the conceptual approach and manuscript preparation; M. K. provided feedback and critical revision of the manuscript and helped with data interpretation and manuscript writing; J. S. supervised the data collection, provided critical review of the manuscript and helped with data interpretation and manuscript preparation. None of the authors declared a conflict of interest. The INMA study was funded by grants from Instituto de Salud Carlos III (Red INMA G03/176, CB06/02/0041, FIS-FEDER 03/1615, 04/1509, 04/1112, 04/ 1931, 05/1079, 05/1052, 06/1213, 07/0314 and 09/02647, FISS-PI042018, FISS-PI09/02311, FIS-PI06/0867, FIS-PS09/ 00090, FIS-PI041436, FIS- PI081151); the Conselleria de Sanitat Generalitat Valenciana, Generalitat de Catalunya-CIRIT 1999SGR 00241; Obra social Cajastur, Universidad de Oviedo, Department of Health of the Basque Government (2005111093 and 2009111069) and the Provincial Government of Gipuzkoa (DFG06/004 and DFG08/001). The RHEA study was partly supported by a Flight Attendant Medical Research Institute grant (Clinical Investigator Award 072058) and by the EU Integrated Projects NewGeneris, 6th Framework Programme (contract no. FOOD-CT-2005-016320) and HiWATE, 6th Framework Programme (contract no. Food-CT-2006036224). We thank all the participants for their generous collaboration.

\section{References}

1. Barker DJ (1997) The long-term outcome of retarded fetal growth. Clin Obstet Gynecol 40, 853-863.

2. Catov JM, Newman AB, Roberts JM, et al. (2007) Association between infant birth weight and maternal cardiovascular risk factors in the health, aging, and body composition study. Ann Epidemiol 17, 36-43.

3. Smith GD, Sterne J, Tynelius P, et al (2005) Birth weight of offspring and subsequent cardiovascular mortality of the parents. Epidemiology 16, 563-569.

4. Richards M, Hardy R, Kuh D, et al. (2002) Birthweight, postnatal growth and cognitive function in a national UK birth cohort. Int J Epidemiol 31, 342-348.
5. King JC (2006) Maternal obesity, metabolism, and pregnancy outcomes. Annu Rev Nutr 26, 271-291.

6. Mikkelsen TB, Osler M, Orozova-Bekkevold I, et al. (2006) Association between fruit and vegetable consumption and birth weight: a prospective study among 43585 Danish women. Scand J Public Health 34, 616-622.

7. Ramon R, Ballester F, Aguinagalde X, et al. (2009) Fish consumption during pregnancy, prenatal mercury exposure, and anthropometric measures at birth in a prospective motherinfant cohort study in Spain. Am J Clin Nutr 90, 1047-1055.

8. Guldner L, Monfort C, Rouget F, et al. (2007) Maternal fish and shellfish intake and pregnancy outcomes: a prospective cohort study in Brittany, France. Environ Health 6, 33.

9. Olsen SF, Osterdal ML, Salvig JD, et al. (2007) Duration of pregnancy in relation to fish oil supplementation and habitual fish intake: a randomised clinical trial with fish oil. Eur J Clin Nutr 61, 976-985.

10. Mendez MA, Plana E, Guxens M, et al. (2010) Seafood consumption in pregnancy and infant size at birth: results from a prospective Spanish cohort. J Epidemiol Community Health 64, 216-222.

11. Olsen SF \& Secher NJ (2002) Low consumption of seafood in early pregnancy as a risk factor for preterm delivery: prospective cohort study. BMJ 324, 447.

12. Halldorsson TI, Meltzer HM, Thorsdottir I, et al. (2007) Is high consumption of fatty fish during pregnancy a risk factor for fetal growth retardation? A study of 44824 Danish pregnant women. Am J Epidemiol 166, 687-696.

13. Ramon R, Ballester F, Iniguez C, et al. (2009) Vegetable but not fruit intake during pregnancy is associated with newborn anthropometric measures. J Nutr 139, 561-567.

14. Mannion CA, Gray-Donald K \& Koski KG (2006) Association of low intake of milk and vitamin D during pregnancy with decreased birth weight. CMAJ 174, 1273-1277.

15. Scholl TO, Chen X, Sims M, et al. (2006) Vitamin E: maternal concentrations are associated with fetal growth. Am J Clin Nutr 84, 1442-1448.

16. Lagiou P, Mucci L, Tamimi R, et al. (2005) Micronutrient intake during pregnancy in relation to birth size. Eur $J$ Nutr 44, 52-59.

17. Jacobs DR Jr \& Steffen LM (2003) Nutrients, foods, and dietary patterns as exposures in research: a framework for food synergy. Am J Clin Nutr 78, 508S-5013S.

18. Knudsen VK, Orozova-Bekkevold IM, Mikkelsen TB, et al. (2008) Major dietary patterns in pregnancy and fetal growth. Eur J Clin Nutr 62, 463-470.

19. Mikkelsen TB, Osterdal ML, Knudsen VK, et al. (2008) Association between a Mediterranean-type diet and risk of preterm birth among Danish women: a prospective cohort study. Acta Obstet Gynecol Scand 87, 325-330.

20. Rodriguez-Bernal CL, Rebagliato M, Iniguez C, et al. (2010) Diet quality in early pregnancy and its effects on fetal growth outcomes: the Infancia y Medio Ambiente (Childhood and Environment) Mother and Child Cohort Study in Spain. Am J Clin Nutr 91, 1659-1666.

21. Trichopoulou A \& Lagiou P (1997) Healthy traditional Mediterranean diet: an expression of culture, history, and lifestyle. Nutr Rev 55, 383-389.

22. Ribas-Fito N, Ramon R, Ballester F, et al. (2006) Child health and the environment: the INMA Spanish Study. Paediatr Perinat Epidemiol 20, 403-410.

23. Chatzi L, Plana E, Daraki V, et al. (2009) Metabolic syndrome in early pregnancy and risk of preterm birth. Am J Epidemiol 170, 829-836.

24. Davey GK, Spencer EA, Appleby PN, et al. (2003) EPICOxford: lifestyle characteristics and nutrient intakes in a 
cohort of 33883 meat-eaters and 31546 non meat-eaters in the UK. Public Health Nutr 6, 259-269.

25. Willett WC, Sampson L, Stampfer MJ, et al. (1985) Reproducibility and validity of a semiquantitative food frequency questionnaire. Am J Epidemiol 122, 51-65.

26. Vioque J, Weinbrenner T, Asensio L, et al. (2007) Plasma concentrations of carotenoids and vitamin $\mathrm{C}$ are better correlated with dietary intake in normal weight than overweight and obese elderly subjects. Br J Nutr 97, 977-986.

27. Trichopoulou A, Costacou T, Bamia C, et al. (2003) Adherence to a Mediterranean diet and survival in a Greek population. $N$ Engl J Med 348, 2599-2608.

28. Chatzi L, Torrent M, Romieu I, et al. (2008) Mediterranean diet in pregnancy is protective for wheeze and atopy in childhood. Thorax 63, 507-513.

29. Westerway SC, Davison A \& Cowell S (2000) Ultrasonic fetal measurements: new Australian standards for the new millennium. Aust N Z J Obstet Gynaecol 40, 297-302.

30. Blair EM, Liu Y, de Klerk NH, et al. (2005) Optimal fetal growth for the Caucasian singleton and assessment of appropriateness of fetal growth: an analysis of a total population perinatal database. BMC Pediatr 5, 13

31. Mamelle N, Cochet V \& Claris O (2001) Definition of fetal growth restriction according to constitutional growth potential. Biol Neonate 80, 277-285.

32. Domingo-Salvany A, Regidor E, Alonso J, et al. (2000) [Proposal for a social class measure. Working Group of the Spanish Society of Epidemiology and the Spanish Society of Family and Community Medicine]. Aten Primaria 25, 350-363.

33. Willet WC \& Stampfer MJ (1986) Total energy intake: implications for epidemiologyic analyses. Am J Epidemiol 124, $17-27$.

34. Hu FB (2002) Dietary pattern analysis: a new direction in nutritional epidemiology. Curr Opin Lipidol 13, 3-9.

35. Willett WC, Sacks F, Trichopoulou A, et al. (1995) Mediterranean diet pyramid: a cultural model for healthy eating. $\mathrm{Am}$ J Clin Nutr 61, 1402S-1406S.
36. Bach A, Serra-Majem L, Carrasco JL, et al. (2006) The use of indexes evaluating the adherence to the Mediterranean diet in epidemiological studies: a review. Public Health Nutr 9 , $132-146$

37. England LJ, Kendrick JS, Wilson HG, et al. (2001) Effects of smoking reduction during pregnancy on the birth weight of term infants. Am J Epidemiol 154, 694-701.

38. Suzuki K, Tanaka T, Kondo N, et al. (2008) Is maternal smoking during early pregnancy a risk factor for all low birth weight infants? J Epidemiol 18, 89-96.

39. Vardavas CI, Chatzi L, Patelarou E, et al. (2010) Smoking and smoking cessation during early pregnancy and its effect on adverse pregnancy outcomes and fetal growth. Eur J Pediatr 169, 741-748.

40. Windham GC, Eaton A \& Hopkins B (1999) Evidence for an association between environmental tobacco smoke exposure and birthweight: a meta-analysis and new data. Paediatr Perinat Epidemiol 13, 35-57.

41. Wang X, Zuckerman B, Pearson C, et al. (2002) Maternal cigarette smoking, metabolic gene polymorphism, and infant birth weight. JAMA 287, 195-202.

42. Palaniappan U, Jacobs Starkey L, O'Loughlin J, et al. (2001) Fruit and vegetable consumption is lower and saturated fat intake is higher among Canadians reporting smoking. J Nutr 131, 1952-1958.

43. Fletcher RH \& Fairfield KM (2002) Vitamins for chronic disease prevention in adults: clinical applications. JAMA $\mathbf{2 8 7}$, 3127-3129.

44. Serra-Majem L, Ribas L, Garcia A, et al. (2003) Nutrient adequacy and Mediterranean diet in Spanish school children and adolescents. Eur J Clin Nutr 57, Suppl. 1, S35-S39.

45. Abrams SA (2007) In utero physiology: role in nutrient delivery and fetal development for calcium, phosphorus, and vitamin D. Am J Clin Nutr 85, 604S-607S.

46. Meltzer HM, Brantsaeter AL, Ydersbond TA, et al. (2008) Methodological challenges when monitoring the diet of pregnant women in a large study: experiences from the Norwegian Mother and Child Cohort Study (MoBa). Matern Child Nutr 4, 14-27. 